RESEARCH NOTE

\section{The Need for an Integrated Approach to the Taxonomy of Neotropical Simuliidae, with Special Reference to Larval Colour}

\section{S Luz/ ${ }^{+}$, AJ Shelley, M Maia-Herzog*}

Department of Entomology, British Museum (Natural History), Cromwell Road, London, SW7 5BD, U.K.

*Departamento de Entomologia, Instituto Oswaldo Cruz, 21045-900 Rio de Janeiro, RJ, Brasil

Key words: Neotropical Simuliidae - taxonomymorphology- cytology

The taxonomy and identification of Simuliidae, a medically important family of Diptera with a worldwide distribution, is largely based on the morphology of adult flies and their pupae and larvae. Amongst the major identification characters used in the identification of morphospecies (species that are able to be identified on morphological characters) is the coloration of adults and larvae and this character has been widely used in both local and regional identification keys in the Afrotropical region [P Freeman \& B de Meillon 1953 Simuliidae of the Ethiopian region, London, British Museum (Natural History), 74 pp]. A taxonomic study of the larvae of West African Simuliidae (Diptera: Nematocera) with comments on the morphology of the larval black-fly head was developed by RW Crosskey (1960 Bul BMNH Entomol 10: 1-74). In the Neotropical region adult coloration and larval head patterns were used as important characters (S Coscarón 1991 Insecta Diptera 2 Simuliidae. Fauna de agua dulce de la República Argentina 304 pp.) and both head pattern and coloration of larvae were used for species recognition in the Santiago onchocerciasis focus of Ecuador, although variations were detected in these characters in some species (AJ Shelley et al. 1989 Bul BMNH Entomol 58: 79-130).

${ }^{+}$Corresponding author. Fax: +55-21-290.9339

Received 5 February 1996

Accepted 15 July 1996
The examination of polytene chromosomes from the silk glands of larvae for the identification of cytospecies (morphologically identical species that are only distinguishable on chromosomal banding patterns) in complexes of vectors of onchocerciasis in Latin America has led to more detailed examination of the morphological characters of larvae. Using a combination of head pattern and body coloration in larvae some of the cytotypes of the 12 recorded for the Simulium metallicum complex, which contains vector cytotypes of human onchocerciasis in Mexico, Guatemala and Venezuela, could be distinguished from one another although geographical variation in these characters was thought to exist (J Conn et al. 1989 Can J Zool 67: 1217-1245, AL Millest 1990 Bul Entomol Res 80: 191-194).

The head pattern character had previously been used in the Santiago onchocerciasis focus of Ecuador to distinguish larvae of the $S$. exiguum complex, the primary vector of the disease there, from the sympatric non vector species $S$. gonzalezi. Shelley et al. (loc. cit.) found that $S$. gonzalezi always had a negative head pattern (dark areas with pale spots on cephalic apotome) while in the $S$. exiguum complex this condition was rare, the normal pattern being positive (dark spots on cephalic apotome). In order to further test the validity of this interspecific character samples of the $S$. exiguum complex from localities both within and outside the onchocerciasis focus were cytotyped and scored for head pattern (M Charalambous et al. 1996 Bul Entomol Res in press). Variations from positive to negative were found in the Cayapa and Quevedo cytotypes of $S$. exiguum to a varying degree in each locality, thereby negating head spot pattern as the main character for separating $S$. exiguum from $S$. gonzalezi. This would be of major significance in any future control campaign using larvicides since it will be impossible to accurately determine species distribution and target only the vector $S$. exiguum.

Integrated morphological and cytological studies on $S$. exiguum cytotypes in Ecuador also revealed that the character of larval colour varied in the Aguarico cytotype. In this form a sex-linked colour dimorphism (green and brown) was observed. Similar variations of brown, green, blue or pink bands have been observed in the primary lowland vector species complex $S$. guianense in the Amazonia focus of Brazil and preliminary indications are that one of the colours may be sex linked and that coloration is probably not cytotype specific (M Charalambous et al. 1996 Med Vet Entomol in press).

An integrated study of the primary vectors of onchocerciasis in lowland areas of the Amazonia 
focus of Brazil, $S$. roraimense and the $S$. oyapockense complex, has provided preliminary evidence for the failure of colour variation as a sound interspecific morphological character. In these species, which both only have a positive head pattern, colour variations include pure white to white with grey, brown or green bands in both species. Studies continue to assess the variation in different populations of $S$. roraimense and cytotypes of the $S$. oyapockense species complex, taking into account the added criterion of larval diet which is thought to also affect coloration.

In conclusion, it is necessary to obtain large samples of larvae for both morphotaxonomic and cytotaxonomic studies to determine whether head pattern and body coloration may be a locally important interspecific character or not, since they are now known to be too variable for use over the wide species range of several vectors of onchocerciasis in Latin America. 